\title{
Qualidade fisiológica e sanitária de sementes de feijoeiro (Phaseolus vulgaris L.) provenientes do estado de Goiás
}

\author{
Physiological and sanity seed quality of common beans \\ (Phaseolus vulgaris L.) from Goias state
}

\author{
Gilvânia Campos Silva ${ }^{1 *}$; Delineide Pereira Gomes ${ }^{2}$; \\ Adriana Zanin Kronka ${ }^{3}$; Myrna Hilal Moraes ${ }^{4}$
}

\section{Resumo}

\begin{abstract}
O feijoeiro comum (Phaseolus vulgaris L.) é uma fabacea bastante difundida em todo território nacional. A baixa qualidade de suas sementes representa uma das principais causas de baixa produtividade nas lavouras de feijão no Brasil. O objetivo deste trabalho foi avaliar a qualidade fisiológica e sanitária de sementes de nove cultivares de feijão provenientes do Estado de Goiás. A qualidade fisiológica das sementes foi avaliada através dos testes de germinação e vigor, e a análise sanitária, através dos métodos de papel de filtro, para verificar a ocorrência de fungos em geral, e do método de Koch e Menten, para a avaliação de Sclerotinia sclerotiorum (Lib) de Bary. As cultivares que tiveram os melhores desempenhos nos testes fisiológicos foram Xamego, BRS Radiante, Bambu e Pérola. As cultivares Jalo Precoce, Roxo 90, Corrente e Aporé apresentaram baixos índices de vigor e germinação de plântulas normais, além de apresentarem os maiores índices de sementes mortas. Quanto à sanidade, verificou-se a incidência dos fungos Fusarium sp., Aspergillus spp., Penicillium sp., Phoma sp., Rhizopus sp. e Botrytis sp.

Palavras-chave: Vigor, germinação, patologia de sementes e feijão
\end{abstract}

\begin{abstract}
The common beans (Phaseolus vulgaris L.) is a fabacea sufficiently spread out in all domestic territory. However, the quality of its seeds represents one of the main causes of low productivity in the beans farmings in Brazil. The objective of this work was to evaluate physiological and sanitary seed qualities of eleven bean cultivars. The physiological seed quality was evaluated trough standard germination and vigor tests. The sanitary seed quality was evaluated through two tests: blotter test was employed to evaluate fungi incidence and "Koch \& Menten" method was employed to observe Sclerotinia sclerotiorum (Lib) de Bary occurrence. Xamego, BRS Valente, Bambu and Pérola had the best results of physiological tests. Jalo Precoce, Roxo 90, Corrente and Aporé had no good results of vigor and germination, besides presenting the lowest indices of died seeds. Fusarium sp., Aspergillus spp., Penicillium sp., Phoma sp., Rhizopus sp. and Botrytis sp. were the fungi detected in the sanity tests.

Key words: Vigor, germination, seed pathology
\end{abstract}

1 Bióloga, Mestre em Agroecologia da Universidade Estadual do Maranhão - UEMA. E-mail: gilvaniacampos@ig.com.br.

2 Agrônoma, Mestranda em Produção e Tecnologia de Sementes - FCAV/UNESP, e-mail: agroneide@hotmail.com.

3 Profa. Dra. do Departamento de Fitossanidade, Engenharia Rural e Solos - UNESP, e-mail:azkronka@yahoo.com.br

4 Mestre em Agroecologia da UEMA.

* Autor para correspondência

Recebido para publicação 15/01/07 Aprovado em 28/08/07 


\section{Introdução}

O feijoeiro comum (Phaseolus vulgaris L.) é a espécie mais cultivada entre as demais do gênero Phaseolus e o seu cultivo está bastante difundido em todo território nacional, no sistema solteiro ou consorciado com outras culturas. É reconhecida como cultura de subsistência em pequenas propriedades e seus grãos constituem importante fonte de proteínas e calorias na dieta humana (UFPEL; FAEM, 2005).

A qualidade das sementes pode ser definida como sendo o somatório de todos os atributos genéticos, físicos, fisiológicos e sanitários que afetam sua capacidade de desempenhar funções vitais, caracterizada pela sua germinação, seu vigor e sua produtividade (POPINIGIS, 1985).

A associação de microorganismos com sementes é de fundamental importância devido aos danos que eles podem provocar às plantas oriundas destas, além de afetar a quantidade e a qualidade do produto final. Estruturas do patógeno presentes nas sementes constituem o inóculo primário para o desenvolvimento de epidemias e este inóculo fica viável por um bom período, quando estas sementes são armazenadas (MACHADO, 1988).

A má qualidade sanitária tem influência na qualidade da semente, com reflexos negativos da cultura no campo, podendo ter efeito na germinação, no vigor e na produtividade, por causar morte da semente, redução do "stand" e doença das plantas (FREITAS, 2005).

O uso de sementes com elevado padrão de sanidade é uma das principais medidas de controle de doenças. Portanto, é recomendável aliar métodos específicos, sensíveis e eficientes, para a detecção de patógenos em sementes e testes fisiológicos na avaliação da qualidade das sementes.

O objetivo do presente trabalho foi a avaliação fisiológica e sanitária de sementes de cultivares de feijoeiro.

\section{Material e Métodos}

O trabalho foi desenvolvido nos laboratórios de Patologia de Sementes e de Análise de Sementes, da Universidade Estadual do Maranhão (UEMA), em São Luís, Maranhão.

As sementes de nove cultivares de feijão (Aporé, Roxo 90, Corrente, Pérola, Bambu, Xamego, Jalo Precoce, BRS Radiante e BRS Talismã) provenientes do Estado de Goiás, foram submetidas a testes de sanidade e de qualidade fisiológica.

\section{Avaliação da qualidade fisiológica}

Para a avaliação da qualidade fisiológica, as sementes foram submetidas aos testes de vigor e germinação, descritos a seguir, de acordo com as Regras para Análise de Sementes (BRASIL, 1992).

Teste padrão de germinação: o teste foi realizado usando-se areia previamente peneirada, lavada e esterilizada como substrato. Foram semeadas em areia, em caixas plásticas, 4 repetições de 100 sementes previamente umedecidas para cada cultivar. A avaliação foi realizada no $9^{\circ}$ dia, através da contagem de plântulas normais, plântulas anormais, plântulas infectadas e sementes mortas (não germinadas). Os resultados foram expressos em porcentagem (\%).

Teste de vigor: o vigor das sementes foi avaliado através da primeira contagem do teste de germinação em areia, realizada no $5^{\circ}$ dia. O resultado foi expresso em porcentagem de plântulas normais.

Para os dois testes, o delineamento experimental utilizado foi o inteiramente casualizado com 4 repetições. Os dados foram transformados em arc sen $\sqrt{\%}$ e submetidos à análise de variância pelo teste F. As médias foram comparadas pelo teste de Tukey, a $5 \%$ de probabilidade.

\section{Avaliação da qualidade sanitária}

As sementes de cada cultivar foram submetidas ao teste de sanidade para verificar a incidência de 
patógenos, através do método de papel de filtro (Blotter test), de acordo com Lucca Filho (1985). O teste consistiu em se colocar três discos de papel de filtro pré-umedecidos em água destilada, em placas de Petri de plástico (diâmetro de $9 \mathrm{~cm}$ ). Em cada placa, foram distribuídas dez sementes equidistantes entre si. Foram feitas 4 repetições de 100 sementes, totalizando 400 sementes por cultivar. As sementes foram incubadas à temperatura de $22^{\circ} \mathrm{C}$ e fotoperíodo de 12 horas sob luz NUV (comprimento de onda entre 320 a $400 \mathrm{~nm}$ ), durante 7 dias. Para a detecção de Sclerotinia sclerotiorum, foi utilizada a metodologia de Koch e Menten (2000), onde as sementes foram incubadas a $15^{\circ} \mathrm{C}$ e escuro contínuo, durante 15 dias. A avaliação do teste foi realizada após os referidos períodos de incubação, examinando-se as sementes, individualmente, com o auxílio de um microscópio estereoscópico. A incidência de fungos foi obtida pela média das 4 repetições das sementes de cada cultivar após o período de incubação.

\section{Resultados e Discussão}

Os resultados da qualidade fisiológica das cultivares de feijão estão apresentados na Tabela 1.

Tabela 1. Qualidade fisiológica de sementes de cultivares de feijoeiro provenientes do Estado de Goiás.

\begin{tabular}{lccccc}
\hline Cultivar & Vigor (\%) & \multicolumn{4}{c}{ Germinação (\%) } \\
\cline { 3 - 6 } & $21,09 \mathrm{bc} *$ & Norntulas & $\begin{array}{c}\text { Plântulas } \\
\text { Anormais }\end{array}$ & $\begin{array}{c}\text { Plântulas } \\
\text { Infectadas }\end{array}$ & $\begin{array}{c}\text { Sementes } \\
\text { mortas }\end{array}$ \\
\hline Aporé & $28,46 \mathrm{bc}$ & $7,00 \mathrm{~b}$ & $10,98 \mathrm{a}$ & $55,08 \mathrm{c}$ \\
Roxo 90 & $7,43 \mathrm{c}$ & $23,00 \mathrm{c}$ & $5,18 \mathrm{~b}$ & $2,00 \mathrm{c}$ & $70,45 \mathrm{ab}$ \\
Corrente & $9,93 \mathrm{c}$ & $16,23 \mathrm{c}$ & $3,29 \mathrm{bc}$ & $5,74 \mathrm{~b}$ & $76,24 \mathrm{ab}$ \\
Pérola & $74,00 \mathrm{a}$ & $75,43 \mathrm{a}$ & $3,92 \mathrm{c}$ & $0,7 \mathrm{~d}$ & $21,97 \mathrm{bc}$ \\
Bambu & $69,38 \mathrm{a}$ & $74,35 \mathrm{a}$ & $6,02 \mathrm{~b}$ & $1,0 \mathrm{~cd}$ & $19,34 \mathrm{bc}$ \\
Xamego & $84,45 \mathrm{a}$ & $86,09 \mathrm{a}$ & $1,72 \mathrm{c}$ & $0,7 \mathrm{~d}$ & $12,93 \mathrm{bc}$ \\
Jalo Precoce & $17,23 \mathrm{bc}$ & $3,37 \mathrm{~d}$ & $3,38 \mathrm{c}$ & $1,0 \mathrm{~cd}$ & $93,57 \mathrm{a}$ \\
BRS Radiante & $58,35 \mathrm{a}$ & $56,25 \mathrm{~b}$ & $4,56 \mathrm{bc}$ & $0,5 \mathrm{~cd}$ & $39,50 \mathrm{c}$ \\
BRS Talismã & $33,23 \mathrm{ab}$ & $45,07 \mathrm{c}$ & $12,30 \mathrm{a}$ & $7,11 \mathrm{ab}$ & $36,61 \mathrm{c}$ \\
\hline CV (\%) & 23,39 & 17,45 & 23,65 & 06,44 & 21,11 \\
\hline
\end{tabular}

*Médias seguidas de mesma letra nas colunas não diferem entre si (Tukey a 5\% de probabilidade).

As cultivares Xamego, BRS Radiante, Bambu e Pérola mostraram melhor qualidade fisiológica das sementes, tendo apresentado um bom desempenho no teste de vigor e resultando nos maiores índices de plântulas normais no teste de germinação. Dentre estas cultivares com melhor desempenho, BRS Radiante também se destacou por não apresentar plântulas infectadas. Por outro lado, Aporé, Jalo Precoce, Corrente e Roxo 90 foram cultivares que resultaram em menor porcentagem de plântulas normais e que tiveram os piores resultados no teste de vigor. As demais cultivares foram intermediárias a esses dois grupos. 
Os resultados obtidos podem ser explicados por diversos fatores. Segundo Carvalho e Nakagawa (2000), a qualidade fisiológica das sementes pode ser influenciada, em maior ou menor grau, pelo tamanho da semente, potencial de armazenamento, condições sanitárias da semente e constituição física e genética, $o$ que provavelmente resultou em diferenças significativas entre elas, com relação aos testes conduzidos.

Apesar do desempenho das cultivares Bambu e Pérola, de acordo com as Normas Técnicas para Produção de Sementes (NTPS, 1999) apenas as cultivares Xamego e BRS Radiante estariam aptas, do ponto de vista fisiológico, a serem cultivadas, por apresentarem germinação acima do padrão recomendado para sementes de feijão, que é de $80 \%$.

Os resultados da análise sanitária das sementes de feijão são mostrados na Tabela 2. Observou-se que as cultivares analisadas apresentaram incidência de Aspergillus spp., variando de 5\%, para Bambu, até 50 $\%$, para BRS Radiante; e Penicillium sp., variando de $0,5 \%$, para Corrente, até $26 \%$, para Roxo 90.

Tabela 2. Incidência de fungos em sementes de cultivares de feijoeiro provenientes do Estado de Goiás.

\begin{tabular}{llllllllll} 
Fungos & Aporé & $\begin{array}{l}\text { Roxo } \\
\mathbf{9 0}\end{array}$ & Corrente & Pérola & Bambu & Xamego & $\begin{array}{l}\text { Jalo } \\
\text { Precoce }\end{array}$ & $\begin{array}{l}\text { BRS } \\
\text { Radiante }\end{array}$ & $\begin{array}{l}\text { BRS } \\
\text { Talismã }\end{array}$ \\
\hline Aspergillus spp. & 20 & 31 & 12 & 17 & 5 & 10 & 39 & 50 & 17 \\
Penicillium sp. & 1 & 26 & 0,5 & 0 & 16 & 15 & 20 & 3 & 1 \\
Botrytis sp. & 0 & 3 & 3 & 1 & 0 & 10 & 3 & 1 & 21 \\
Rhizopus sp. & 0,25 & 0 & 0,5 & 0 & 30 & 0 & 3 & 6 & 0 \\
Fusarium sp. & 0 & 3 & 8 & 7 & 0 & 7 & 2 & 4 & 2 \\
Phoma sp. & 0 & 0 & 0 & 0 & 0 & 0 & 0 & 1 & 0 \\
S. sclerotiorum & 0,3 & 0 & 0,8 & 0 & 0 & 0 & 0,3 & 0 & 0 \\
\hline
\end{tabular}

Com o advento da irrigação, a cultura do feijão passou a ter plantio contínuo, possibilitando que um complexo de doenças causadas por patógenos veiculados pelo solo adquirisse grande importância. Dentre estas doenças, tem-se as fusarioses (murcha de fusarium e podridão radicular, causada por Fusarium sp.) e o mofo branco, causado por Sclerotinia sclerotiorum. No presente estudo, foi observada a incidência de Fusarium sp. em 8 cultivares avaliadas. Trata-se de uma constatação preocupante, pois este fungo tem a capacidade de sobreviver no solo, na forma de clamidósporos, e é disseminado através de sementes contaminadas e/ ou infectadas. Com relação a S. sclerotiorum, embora tenha ocorrido em baixas taxas, as três cultivares nas quais foram observadas devem ser evitadas por se tratar de um patógeno com grande poder destrutivo e capacidade de sobrevivência por muitos anos, através dos escleródios (HALL; STEADMAN, 1994).

O gênero Rhizopus sp., apesar de não possuir importância econômica nas sementes, pode, como contaminante, dificultar a detecção de patógenos, por cobrir as sementes com rápido crescimento (TORRES; BRINGEL, 2005).

Faiad, Ramos e Wetzel (2004), que utilizaram a mesma metodologia para a detecção de fungos, também encontraram fungos patogênicos como Fusarium sp. e Phoma sp. em sementes de feijão armazenadas e longo prazo.

Espécies do gênero Aspergillus foram observadas em sementes de feijão procedentes do Estado da Paraíba por Benício et al. (2005). Coutinho, Araújo e Araújo (2004) em feijão comum, e Torres e Bringel (2005) em feijão macassar, também constataram uma 
micoflora formada por Aspergillus spp. e Penicillium spp. Nem todas as espécies de fungos são toxigênicas, mas sabe-se que mais de 300 espécies podem produzir algum tipo de toxina. Dentre as principais micotoxinas encontradas em produtos alimentícios e grãos, têm-se a aflatoxina e a ocratoxina, produzidas por fungos de armazenamento dos gêneros Aspergillus e Penicillium, sendo os produtos mais afetados os cereais e fabaceas, e entre estas, o feijão (ENCAL, 2005).

Lobo Júnior e Cobucci (2004), que também trabalharam com a cultivar Pérola, constataram que as sementes tratadas com Fludioxanil + Difeconazole (200 mL $+34 \mathrm{~mL} / 100 \mathrm{~kg}$ de sementes) deram origem a plantas com $5 \%$ do sistema radicular com sintomas de podridão por Fusarium solani e Rhizoctonia solani. Lima et al. (2004), que trabalharam com as cultivares Diamante Negro, Pérola e Jalo Precoce, relatam que $100 \%$ destas apresentaram contaminação pelo gênero Fusarium e também pelos fungos de armazenamento.

\section{Conclusão}

Comparando-se os resultados obtidos para a qualidade fisiológica e a incidência de fungos, observou-se que, em geral, os altos índices de vigor dos genótipos Bambu e BRS Radiante podem estar relacionados com a baixa incidência de fungos e principalmente, com a ausência de Fusarium sp. e S. sclerotiorum. Os baixos percentuais de vigor e os altos índices de sementes mortas dos genótipos Corrente e Jalo Precoce podem estar associados pela presença de praticamente todos os fungos (com exceção de Phoma sp.), principalmente, pela incidência dos principais patógenos do feijoeiro (Fusarium sp. e Sclerotinia sclerotiorum).

\section{Referências}

BENÍCIO, V.; ARAÚJO, E.; SOUTO, F. M.; BENICIO, M. J.; FELISMINO, D. C. Identificação e características culturais de espécies do gênero Aspergillus isoladas de sementes de feijão no Estado da Paraíba. Disponível em: $<$ http://www.scielo.br/scielo.php?pid=S010041582003000200011\&script=sci_arttext\&tlng=pt-> Acesso em: 01 abr. 2005.

BRASIL. Ministério da Agricultura. Secretaria Nacional de Defesa Agropecuária. Regras para Análise de Sementes. Brasília: SNDP/DNDB/CLAV, 1992.

CARVALHO, N. M.; NAKAGAWA, J. Sementes: ciência, tecnologia e produção. 5. ed. Campinas: Fundação Cargill, 2000.

COUTINHO, W. M.; ARAÚJO, E.; ARAÚJO, D. L. Micoflora de sementes de feijão tratadas com extrato de sucupira e com os fungicidas Benomyl e Captan. In: SIMPÓSIO BRASILEIRO DE PATOLOGIA DE SEMENTES, 8., 2004, João Pessoa, PB. Palestras e Resumos... João Pessoa, 2004. p.167.

ENCAL. Micotoxinas. Disponível em: <http:// www.scielo.br/scielo.php? pid=S 0100 $41582003000200011 \&$ script $=$ sci_arttext\&tlng $=$ pt- $>$. Acesso em: 01 abr. 2005.

FAIAD, M. G. R.; RAMOS, V. R.; WETZEL, M. M. V. S. Fungos associados a sementes de feijão (Phaseolus vulgaris L.) armazenadas a longo prazo. In: SIMPÓSIO BRASILEIRO DE PATOLOGIA DE SEMENTES, 8., 2004, João Pessoa. Palestras e Resumos... João Pessoa, 2004. p. 171.

FREITAS, R.A. Patologia de semente de feijão. Disponível em: <http://orbita.starmedia.com/ $\sim$ fitopatologia/patofeijao.htm>. Acesso: 17 abr. 2005.

HALL, R.; STEADMAN, J. R. White mold. In: HALL, R. (Ed.) Compendium of bean diseases. 2. ed. Saint Paul: APS Press, 1994. p. 28-29.

KOCH, E. F. A.; MENTEN, J. O. M. Método alternativo para detecção de Sclerotinia sclerotiorum em sementes de feijoeiro. Summa Phytopathologica, Jaboticabal, v. 26, n. 2, p. 276-279, 2000.

LIMA, B.; REY, M. S.; FARIAS, C. R. J. de.; BRANCÃO, M. F.; PIEROBOM, C. R. Qualidade sanitária de sementes de diferentes cultivares de feijão (Phaseolus vulgaris L.). Fitopatologia Brasileira, Brasília, v. 29, p. 182, 2004. 
LOBO JÚNIOR, M.; COBUCCI, T. Efeito da qualidade e do tratamento de sementes no controle de podridões radiculares e podridão do feijoeiro comum cv. Pérola. In: SIMPÓSIO BRASILEIRO DE PATOLOGIA DE SEMENTES, 8., 2004, João Pessoa. Palestras e Resumos... João Pessoa, 2004. p. 183.

LUCCA FILHO, C. A. Importância da sanidade na produção de sementes de alta qualidade. Revista Brasileira de Sementes, Pelotas, v. 7, n. 1, p. 113-123, 1985.

MACHADO, J. C. Patologia de sementes: fundamentos e aplicações. Lavras: ESAL/FAEPE, 1988.

MARANHÃO. Comissão Estadual de Sementes e Mudas do Estado do Maranhão - CESM-MA. Normas técnicas para produção de sementes (NTPS). Balsas, 1999.
POPINIGIS, F. Fisiologia da semente. Brasília: Agiplan, 1985.

TORRES, S.B.; BRINGEL, J.M.M. Avaliação da qualidade sanitária e fisiológica de sementes de feijão macassar. Caatinga, Mossoró, v. 18, n. 2, p. 88-92, 2005.

UNIVERSIDADE FEDERAL DE PELOTAS - UFPEL; FACULDADE DE AGRONOMIA ELISEU MACIEL FAEM. Patologia de sementes: feijão. Disponível em: $<$ http://www.ufpel.edu.br/faem/dfs/patologiasementes/

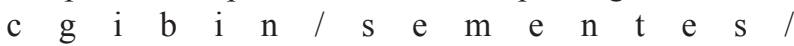
procura.cgi?tipo $=4 \&$ cultura $=8 \&$ tem $=0 \mathrm{k}>$. Acesso em: 22 mar. 2005. 